\title{
Editorial \\ Capturing real-life patient care in psoriatic arthritis and its risks: the challenge of analysing registry data
}

\section{Daniel Aletaha}

Division of Rheumatology, Medical University of Vienna, Vienna, Austria

Corresponding author: Daniel Aletaha, daniel.aletaha@meduniwien.ac.at

Published: 3 June 2009

This article is online at http://arthritis-research.com/content/11/3/112

(c) 2009 BioMed Central Ltd

See related research by Saad et al., http://arthritis-research.com/content/11/2/R52
Arthritis Research \& Therapy 2009, 11:112 (doi:10.1186/ar2694)

\begin{abstract}
Studies based on registries continue to inform us of many relevant issues in the treatment of arthritic conditions and constitute more than just a supplement of clinical trial data. We can learn about long-term aspects of therapies beyond the scope of most clinical trials and about larger-scale toxicity. The downsides need to be considered in the interpretation of the results and include mainly the biases that are inherent when routine clinical practice is just observed and not steered by a protocol. However, using steered protocols in practice not only would facilitate post hoc analyses of clinical effectiveness, but (as we have learned from research in rheumatoid arthritis) can also improve outcomes of our patients.
\end{abstract}

In a recent issue of Arthritis Research \& Therapy, Saad and colleagues [1] present a study on the persistence with antitumour necrosis factor (anti-TNF) therapy in psoriatic arthritis (PsA). And as is the case for most publications that stem from registries, there will be those excited about learning important real-life aspects of our therapies and there will be those critical about it, citing all of the biases of observational studies that can be reviewed in epidemiological textbooks. What unites these different viewpoints is that we have become increasingly aware in the past decades that there are certain things and medical facts that we will never learn from clinical trials, either because they are not able to address them (for example, large-scale toxicity) or because they do not want to address them (for example, comparative efficacy). So what can we learn from the work of Saad and colleagues [1] that we cannot learn from clinical trials?

To start with the most delicate question that can be posed: Which drug is most effective, and which one is least toxic? Usually, this issue is handled with a lot of care, as it is by the authors of the current study, but the general impression remains that infliximab might be inferior in regard to both discontinuation rates for ineffectiveness and toxicity. This conclusion, of course, is challenging, as the analysis of drug retention rates often creates clinical paradoxes, such as the one seen in rheumatoid arthritis (RA), in which methotrexate retention rates seem to have decreased over the years. The reason is that treatment termination, as the natural anchor for analyses of retention rates, at least for lack of effectiveness, has become a moving target [2]. In other words, a switch of anti-TNF to a subsequent regimen can imply that clinical disease activity was unacceptable, but it also includes the clinical philosophy of what is considered 'unacceptable' at the time of observation. For example, a large number of potential spare regimens will lower the bar of intolerable disease activity for treatment changes considerably. New therapeutic options aside, the perception of disease activity (and when it is deemed intolerable) changes over the years.

However, for the comparison of the three TNF inhibitors in this study, we can probably only follow the line of arguments by the authors that several reasons may exist that potentially penalise infliximab in the comparison, with channelling bias being one of them. We therefore might turn around and once again raise our voice and call for comparative clinical trials. Activities from registries like the British Society for Rheumatology Biologics Register, which is sponsored by essentially all of the pharmaceutical companies that are marketing biologic therapies, indicate that there is some hope that one day one or the other of these companies will take the economic risk and engage in a head-to-head trial design of different biologic drugs as well as in strategic trials involving different biologic drugs. In this way, we eventually will be able to learn more from trials than the fact that adding a new therapy to methotrexate has superior efficacy than adding placebo.

However, even then, the need for observational studies would remain, and since comorbidity has been addressed in the

$\mathrm{PsA}=$ psoriatic arthritis $\mathrm{RA}=$ rheumatoid arthritis; $\mathrm{TNF}=$ tumour necrosis factor. 
study by Saad and colleagues, it can serve as a very good example for that: comorbidity usually is neglected and excluded in clinical trials but is omnipresent in real life. Based on the data in this study, the presence or absence of any comorbidity has an impact on the occurrence of adverse events that limit drug continuation. As an additional layer of complexity, chronic arthritis causes comorbidity itself [3,4], emphasising the importance of preventive arthritis care.

In regard to the issue of predictors, it seems to be another paradox that higher disease activity levels were found to better predict retention of TNF inhibitors. The potential bias that these patients were likely to be more refractory can be adjusted for in part, but it is difficult to allow for the clinical 'gut' feelings of rheumatologists, by which most of us would tend to keep a patient on a given therapy if that patient has already improved to some extent since the initiation of the respective treatment. Showing such large amounts of improvement is more likely for someone who started off with higher disease activity than for someone coming from a lower baseline, even if the patients have reached the same unsatisfying endpoint. This might, at least in part, explain this paradox. In RA, therapy steered by an index has become more and more a mainstay of management as it has been shown that it is not the level of improvement that is decisive but rather the disease activity reached $[5,6]$. This helps to develop and advance 'gut' decisions to more objectively informed ones and will likely benefit patients with PsA.

One word on the power of statistical computation, which is also perceivable in this study: the authors have adopted the methodology of looking at all treatment terminations and then at only those terminations for the reason of ineffectiveness and terminated for the reason of toxicity. This was done by censoring patients with treatment terminations other than the reason of interest in the respective subanalysis. For example, if survival of drug effectiveness is analysed, a patient with toxicity-related termination of therapy will be considered to have reached the end of observation without reaching the outcome [7]. In this way, the observed period that was eventfree (that is, no ineffectiveness of the drug) can inform the analysis. In contrast to stratification, this is an effective way to incorporate observations on all patients in the analysis. The dichotomy between ineffectiveness and toxicity as a physician-reported reason for drug discontinuation carries another problem: the issue of adjudication. Some patients clearly will have unacceptable flares, and others clearly will have unacceptable toxicity; however, the vast majority of patients will have some combination of the two, which will hamper a clear adjudication of the reason for withdrawal.

In summary, the study by Saad and colleagues gives us important insights into daily-life use of TNF inhibitors in PsA, a disease that too often is secondary to RA in the scientific and clinical perceptions of rheumatologists. The authors deal effectively with the challenges posed by the type and origin of their data. Although toxicity and effectiveness analyses seem to favour etanercept and adalimumab over infliximab, we need to be cautious unless we have results from randomised comparative trials.

\section{Competing interests}

The author declares that he has no competing interests.

\section{References}

1. Saad AA, Ashcroft DM, Watson KD, Hyrich KL, Noyce PR, Symmons DPM: Persistence with anti-TNF therapies in patients with psoriatic arthritis: observational study from the British Society of Rheumatology Biologics Register. Arthritis Res Ther 2009, 11:R52.

2. Aletaha D, Smolen JS: Threats to validity of observational studies on disease-modifying antirheumatic drug therapies for rheumatoid arthritis: new aspects after the fall of the pyramid and the rise of new therapeutics. Curr Rheumatol Rep 2003, 5:409-412.

3. Pincus T, Callahan LF: Taking mortality in rheumatoid arthritis seriously-predictive markers, socioeconomic status and comorbidity. J Rheumatol 1986, 13:841-845.

4. del Rincon I, Williams K, Stern MP, Freeman GL, Escalante A: High incidence of cardiovascular events in a rheumatoid arthritis cohort not explained by traditional cardiac risk factors. Arthritis Rheum 2001, 44:2737-2745.

5. Aletaha D, Funovits J, Smolen JS: The importance of reporting disease activity states in rheumatoid arthritis clinical trials. Arthritis Rheum 2008, 58:2622-2631.

6. Grigor C, Capell H, Stirling A, McMahon AD, Lock P, Vallance R, Kincaid W, Porter D: Effect of a treatment strategy of tight control for rheumatoid arthritis (the TICORA study): a singleblind randomised controlled trial. Lancet 2004, 364:263-269.

7. Aletaha D, Smolen JS: Effectiveness profiles and dose dependent retention of traditional disease modifying antirheumatic drugs for rheumatoid arthritis. An observational study. J Rheumatol 2002, 29:1631-1638. 